\title{
Experimental Information on the Adsorbed Phase of Water Formed in the Inner Pore of Single-Walled
}

\section{Carbon Nanotube Itself}

Masayasu Nishi, ${ }^{\dagger}$ Takahiro Ohkubo, ${ }^{\dagger},{ }^{*}$ Koki Urita, ${ }^{\ddagger}$ Isamu Moriguchi, ${ }^{\ddagger}$ Yasushige Kuroda ${ }^{\dagger}$

†Department of Chemistry, Graduate School of Natural Science and Technology, Okayama University, 3-1-1 Tsushimanaka, Kita-ku, Okayama 700-8530, Japan

${ }^{\dagger}$ Division of Chemistry and Materials Science, Graduate School of Engineering, Nagasaki

University, 1-14 Bunkyomachi, Nagasaki 852-8521, Japan

${ }^{*}$ To whom correspondence should be addressed. Tel \& Fax: +81-86-251-7843

E-mail: ohkubo@okayama-u.ac.jp 


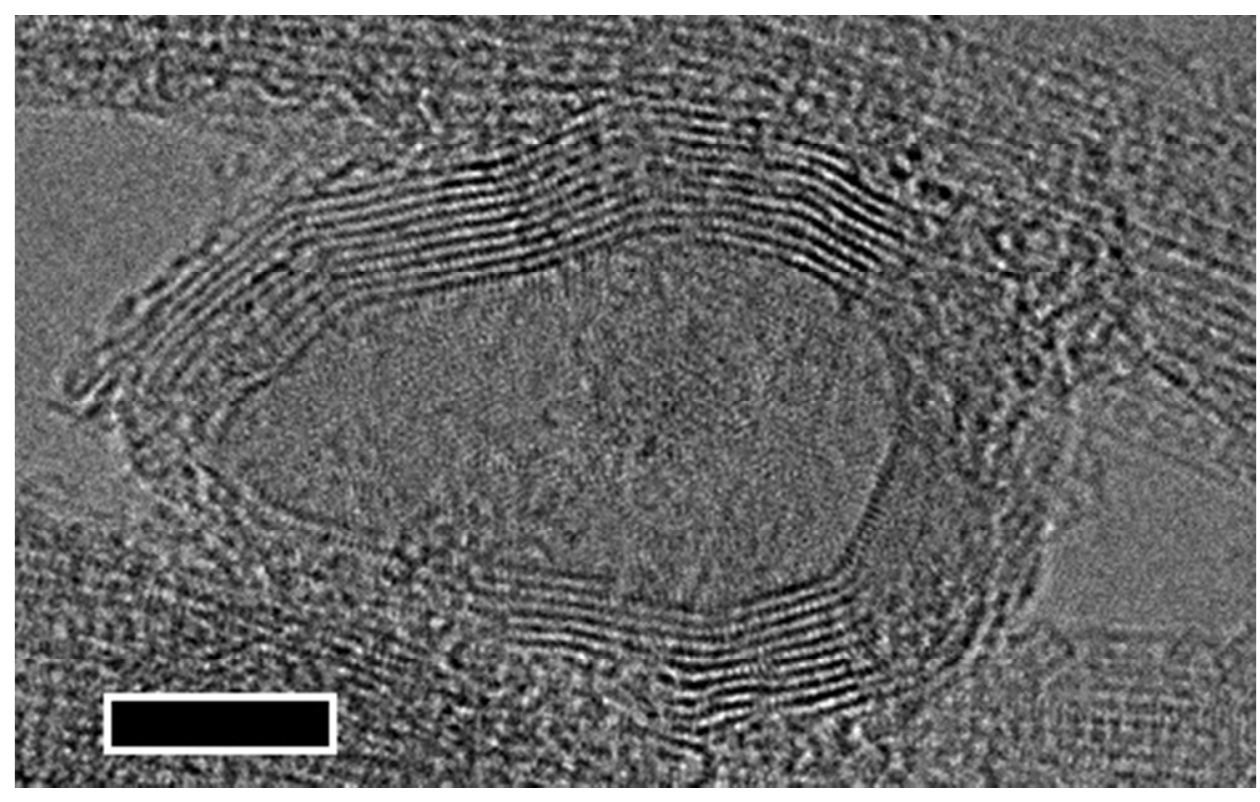

Figure S1. TEM image of Arc-SWCNT. Scale bar: $5 \mathrm{~nm}$. 